\title{
Recognition of Neonatal Hospitalization Cost in an Intensive Care Unit in Greece and the Deviation from Its Current Reimbursement System
}

\author{
Antonia CHRISTOFORIDOU ${ }^{\mathrm{a}}$, Charalambos PLATIS ${ }^{\mathrm{a}, 1}$, Emmanouil ZOULIAS $^{\mathrm{b}}$ and \\ Giannis KARAFYLLIS ${ }^{\mathrm{a}}$

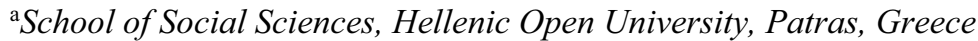 \\ ${ }^{\mathrm{b}}$ Faculty of Nursing, National and Kapodistrian University of Athens, Athens, Greece
}

\begin{abstract}
In this paper efforts have been made to record the actual, real cost of health care services in a Neonatal Intensive Care Unit (N.I.C.U.) of a public hospital. It is well known that, in recent years, the hospitals have been reimbursed with the system of Diagnosis-Related Groups (D.R.G.'s). The purpose of this study is to determine whether the costs according with D.R.G.'s correspond to the actual-real cost, as this is recorded in the N.I.C.U. This cost is called direct cost. Here is a case study of a premature neonate in the intensive care unit (N.I.C.U.). From the outset, the age of pregnancy, the birth weight, the duration of hospitalization in N.I.C.U. and the needs of the newborn in oxygen, medication, as well as nutrition are defined which are very important in shaping the cost. Then, the cost is calculated according to the D.R.G.'s system. By setting three basic diagnoses (I.C.D. -10), we find the D.R.G. which better describes the case, as well as the associated costs. Then, we calculate the direct cost and list all the consumables, exams, staff costs, overheads. Comparing the two results we find that the cost of D.R.G. does not meet the direct cost of hospitalization. There is a significant deviation from the actual real cost, which proves the under-costing of the health services. The D.R.G.'s system leads hospitals to increase their financial deficits and provide degraded quality health services. It is necessary to readjust the D.R.G.'s according to the reality and the redefinition of the hospital's reimbursement system to meet the direct - real cost of the health services offered.
\end{abstract}

Keywords. Neonatal Intensive Care Unit, Cost, Health Services, Premature Newborn, Diagnosis-Related Groups.

\section{Introduction}

In order to determine the cost of the health services offered within the neonatal intensive care unit, we investigated a case study involving a premature new-born at approximately 30 weeks gestation. Based on statistics, his birth weight was estimated at $1200 \mathrm{gr}$ [1]. The minimum time of hospitalization of the new-born is until they reach the 36th week of pregnancy, i.e. about 6 weeks, considering that their body weight then is above $2000 \mathrm{gr}$.

${ }^{1}$ Corresponding Author, Charalambos Platis, School of Social Sciences, Hellenic Open University, Patras, Greece; E-mail: chplatis@gmail.com. 
The assumptions-conditions set for the hospitalization of the new-born are as follows: The new-born remained in the incubator for most of its hospitalization and only in the last 7 days in a simple shake. We thought it was intubated for 1-2 days, then put on N.C.P.A.P. for 5 days and on diffuse oxygen for 2-3 days. The incident was given a parenteral nutrition for 4 days, followed by intestinal feeding with special milk via a nasogastrical feeding catheter for 1-2 days. The rest of the time the new-born was fed normally with feeding bottle, assuming the baby did not breastfeed for various reasons. Due to prematurity [2], the new-born was put into phototherapy for a 24-hour period during hospitalization.

The total days of hospitalization are 42, with 35 corresponding to days of hospitalization in intensive care (N.I.C.U.) while in the last 7 days he was hospitalized in H.D.U. (High-Dependency Unit) for follow-up. The health services the new-born received throughout this period concern:

1. The technological equipment of the unit (respirator, incubator, etc.).

2. Parenteral feeding as mentioned above.

3. Blood Tests.

4. Imaging examinations (X-rays, ultrasounds).

5. Medications. (antibiotics, oxygen, serums, etc.).

6. Consumable materials. Sanitary material (sterile and non-sterile) and all consumable materials e.g. stationery.

7. Overheads. The cost of cleaning, water, electricity, etc.

\section{Cost Determination}

According to the I.C.D.-10 coding system for the costing of this hospitalization, the main diagnosis of admission of the newborn was first determined and then the secondary diagnoses, which characterize the course of his hospitalization. Through the implementation of the hospital reimbursement system (http://kenicd.e-healthnet.gr), the codes of the diagnoses we set were identified:

- Premature birth code icd-10. .p07.3

- New-born respiratory distress code icd-10_.........p22

- Problems feeding new-born code icd-10. p92

- Neonatal jaundice related to prematurity. code icd-10 .p59.0

In connection with these diagnoses, a search was made for the appropriate D.R.G. which is more responsive to this particular incident. This D.R.G. (T23A) includes specified medical operations and a fixed Average Length Of Hospital Stay-A.L.O.S. (35 days) at the corresponding cost (6390 €s). The extra days from A.L.O.S. which gives the selected D.R.G. were charged with the daily hospital cost of the Greek N.H.S. (as stated in the F.E.K. B2315/1.10.2018) and this amount corresponds to $1050 €$ (7days*150euro/day). Therefore, the total amount by which the hospital will be compensated for the hospitalization of the premature new-born amounts to $7440 €$.

Then, the actual costs, as shown by the costing of all those determining the direct health costs, were determined. Direct costs refer to both direct medical and nonmedical costs and include all hospital resources spent during neonatal hospitalization. The direct medical costs are those directly related to the health care provided to the 
new-born and include all those costs incurred in the performance of medical work and nursing care.

The direct medical costs include the technological equipment of the plant, laboratory tests, medicines, consumables, the cost of medical staff and special health care in the N.I.C.U.. The direct non-medical costs are not directly linked to the health care services we provide to premature infants, but no one can overlook its necessity for the full and proper organisation of the health unit. It includes all those costs and expenses that will necessarily be incurred during the hospitalization of the new-born, but not aimed at any health service. Thus, this cost includes the costs associated with the cleaning crew, the laundry company for clothing, the feeding of the premature, but also the water and electricity bills of the unit. After a detailed recording of all these resources and calculating the minimum consumption required, the direct cost within the intensive care unit in the case we are studying, is formed according to the following table:

Table 1. The Cost of Hospitalization in N.I.C.U.

\begin{tabular}{lll}
\hline \multicolumn{1}{c}{ Hospitalization Cost in N.I.C.U. } & \\
\hline Direct Medical Cost & For $\mathbf{3 5}$ days & For $\mathbf{4 2}$ days \\
TYPE & $868.30 €$ & $868.30 €$ \\
Ledical Devices [3] & $1046.21 €$ & $1104.73 €$ \\
Medication Cost [5]-[8] & $384.42 €$ & $384.42 €$ \\
Consumable Materials Cost [9]-[10] & $501.56 €$ & $506.95 €$ \\
Staff Cost [11]-[13] & $4209.10 €$ & $5050.92 €$ \\
Special Health Care [14]-[17] & $174.25 €$ & $174.25 €$ \\
Direct Non-Medical Cost & & \\
TYPE & For 35 days & For $\mathbf{4 2}$ days \\
Hotel Care (Clothing, Nutrition, Cleanness) & $491.85 €$ & $567.10 €$ \\
Overheads (Electric Reference, Water) [18] & $135.44 €$ & $135.44 €$ \\
TOTAL HOSPITALIZATION COST & $\mathbf{7 8 1 1 . 1 3} €$ & $\mathbf{8 7 9 2 . 1 1} €$ \\
\hline
\end{tabular}

\section{Results-Conclusions}

The results are shown aggregated in Table 2:

Table 2. Summary table

\begin{tabular}{ccc}
\hline Cost Estimation Method & 35-Days Hospital Stay & 42-Days Hospital Stay \\
\hline D.R.G. Reimbursement & $6390 €$ & $7440 €$ \\
Direct Cost & $7811.13 €$ & $8792.11 €$ \\
\hline
\end{tabular}

The difference between the direct cost and the reimbursement given by the D.R.G. is approximately $18.2 \%$ for the duration of the 35 days of hospitalization, while this difference is reduced to $15.4 \%$ for the 42 days. This is due to the fact that in the last 7 days of hospitalization, the new-born does not follow any medication and does not need specialized care, while the daily hospital cost in H.D.U. is lower. For this reason, we focused on the cost of 35 days, i.e. the A.L.O.S. given by the D.R.G.. The participation rate of each category in the formation of direct costs, as detailed, is schematically depicted in Figure 1, where it is shown that the largest portion is the cost of staff (nursing - medical). 


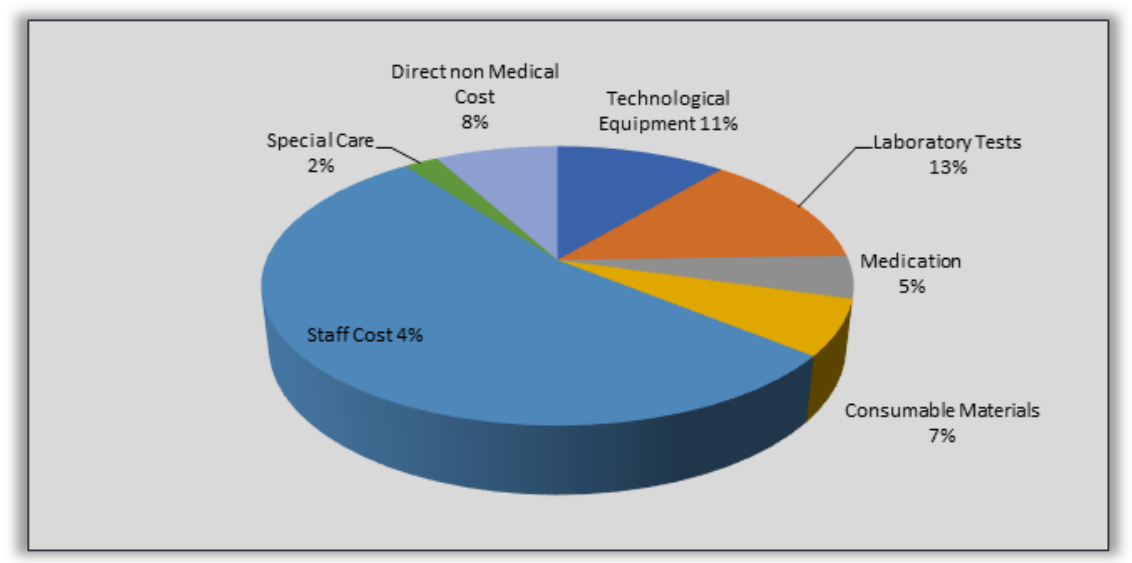

Figure 1. Direct Cost.

From the comparison of the two results, it was found that the costs attributed through the D.R.G do not correspond to reality, which means that, for each incident within the N.I.C.U. the hospital consumes resources, which are not covered by that amount that the insurance institution eventually reimburses. This under-costing of health services (increased costs without the corresponding revenues) leads to the creation of a "deficit" hospital, which does not have necessary materials-medicines, qualitatively degrading the health care and services it provides. These constant difficulties in the execution of nursing and medical work cause professional burnout in the staff and result in a reduction in the efficiency and effectiveness of the hospital. This affects the accessibility of citizens and calls into question the ability of the health system itself to perform its role within society.

This is highlighted in a study carried out in Sao Paulo, Brazil in 2004 [19]. In the research conducted at the time, an attempt was made to estimate the cost of hospitalization of premature infants in the NICU of a tertiary hospital. The result of the investigation of Desgualdo Cl.M., Riera R., Zucchi P. showed that the reimbursement that the insurer returns to the hospital is not proportional to the direct costs spent in N.I.C.U. The deviation between them is significant, with the cost of staff being the one that has the greatest contribution to direct costs.

This under-costing of health services does not only apply to intensive care units. Similar results are highlighted through the research of Kotsalas N. [20] that was done in 2016 and was concerned with the cost of Haemodialysis treatment. In this study, the actual cost of haemodialysis was estimated in two different public hospitals in Greece and then this cost was compared with the corresponding one in England and the USA. It should be noted that the haemodialysis' actual cost, between of the two health units shows an increase of about $20.4 \%$ in the hospital with the less cases of haemodialysis per day. The cost was calculated at $202.1 €$ in the one hospital and, in the other one at 167.86€. The appropriate D.R.G. for haemodialysis" procedure is "Y29A" according to which the amount of $136 €$ is reimbursed to the hospital. There is also in this study, a significant under-costing of health services, with the deviation of the real cost from the corresponding D.R.G.s compensation to range from $48.61 \%$ to $23.42 \%$ (respectively for each hospital).

In conclusion, it can be said that while the implementation of the D.R.Gs system, the hospitals aimed at better administration and organization of health units, improving 
the quality of health services, transparency of procedures, efficiency of the health system [21] and rational use of already limited resources [22], the rough and incomplete design of the system and the horizontal nature of D.R.Gs has led to a significant deviation from the actual cost of health services.

\section{References}

[1] Tokmakidis P. Obstetrics. Third issue. Thesaloniki: TEI Thessalinikis; 1997.

[2] Lissauer T, Clayden G. Modern Paediatrics. Nicosia, Cyprus: Broken Hill Publishers LTD; 2016.

[3] World Health Organization. WHO compendium of innovative health technologies for low-resource settings. Geneva, Switzerland: WHO Document Production Services; 2015.

[4] National Organization for the Provision of Health Services (EOPPYY). Search for Approved EOPYY Products, Medical Specialties and Contracted Providers, Available at: https://eservices.eopyy.gov.gr/eSearchAllMaterial/secure/instructions.xhtml?faces-redirect=true, Accessed August 19, 2019.

[5] Hellenic Infection Society. Guidelines for the diagnosis and empirical treatment of infections. Athens: Focus on Health Ltd; 2007.

[6] LeMone P, Karen B, Bouldoff G. Medical - Surgical Nursing Critical Thinking in Patient Care. New Jersey: Pearson; 2008.

[7] Gargasz A. Neonatal and pediatric parenteral nutrition. AACN Adv Crit Care. 2012 OctDec;23(4):451-64.

[8] Smith A, Glynn AC, Shankar A, McDermott C, McCallion N. A Review of the Parenteral Nutrition Supply Service in an Irish Neonatal Unit. Ir Med J. 2018 Jun 7;111(6):778.

[9] Procurement committee of Ministry of Health. Drug Price Sheets, Available at:, http://84.205.248.47/front.php/simple/listing. Accessed August 23, 2019.

[10] Ministry of Health. Consumable Materials Cost, Available at: http://84.205.248.47/front.php/simple/listing, Accessed 23 august 2019.

[11] Androulaki I. National Health Care System: Danger to the lives of patients gaps in nurses. Available at: https://www.cnn.gr/focus/story/90604/esy-kindynos-gia-tin-zoi-ton-asthenon-ta-kena-se-nosileytes-aud, Accessed August 4, 2019.

[12] Vlachioti E, Dousis E, Matziou V. Safe Staffing in a Pediatric Hospital. Hellenic Journal of Nursing. 2011;50(2):177-184.

[13] Ministry of Finance. Salary Issues, Available at: https://www.minfin.gr/web/31511/nomoi-pouaphoroun-te-misthodosia-demosion-ypallelon, Accessed August 23, 2019.

[14] Chatziioannidis I. Development of a database of indicators of health outcomes and nursing care in a neonatal intensive care unit. Diploma Thesis, University of Macedonia, Thessaloniki: Thessaloniki Psiphida Institutional Repository http://dspace.lib.uom.gr/handle/2159/16996; 2015.

[15] Maragkaki E. Prematurity. Evaluation Of The Risk Factors. Diploma Thesis, National and Kapodistrian University of Athens, Department of Health Sciences, Medical Faculty: EKT Repository http://dx.doi.org/10.12681/eadd/5287; 1995.

[16] Siaxos M. The new payroll for doctors and dentists - Salaries, allowances, on-call time - Detailed tables,

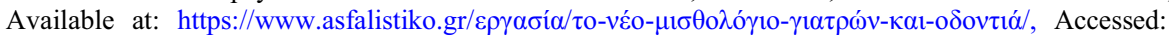
August 23, 2019.

[17] Kadda D. How to cost your products and services. Available at: https://www.capital.gr/epikairotita/1672800/pos-na-kostologisete-ta-proionta-kai-tis-upiresies-sas, Accessed: July 5, 2019.

[18] Kantolian. How much does a kWh cost? Kantoliana.gr. Available at: https://kantoliana.gr/blog/posokostizei-mia-kwh/, Accessed: August 6, 2019.

[19] Desgualdo C, Riera R, Zucchi P. Cost estimate of hospital stays for premature newborns in a public tertiary hospital in Brazil. Clinics (São Paulo, Brazil). 2011;66:1773-7.

[20] Kotsalas. N. Comparative Study of the Cost of Haemodialysis Treatment In Patients with end-stage Renal Disease Between Greece, Great Britain and the USA. 27 (162) Hellenic Health Services Management Association; 2016, 27(162).

[21] Busse R, Geissler A, Quentin W, Wiley M. Diagnosis-Related Groups in Europe Moving towards transparency, efficiency and quality in hospitals. Glasgow: World Health Organization 2011 on behalf of the European Observatory; 2011.

[22] Spyropoulos V. Cost Management. Athens: Kallipos Repository https://repository.kallipos.gr/bitstream/11419/3051/1/02_chapter_16.pdf; 2015. 